\section{Regional Distribution of Neurofibrillary Tangles and Senile Plaques in the Cerebral Cortex of Elderly Patients: A Quantitative Evaluation of a One-Year Autopsy Population from a Geriatric Hospital}

Detailed analyses of the neuropathologic changes in the cerebral cortex of elderly individuals and Alzheimer's disease patients have demonstrated that certain components of the neocortical and hippocampal circuits are likely to be selectively vulnerable. Based on the distribution of neurofibrillary tangles (NFTs) and senile plaques, it has been proposed that a global corticocortical disconnection leads to the loss of integrated functions observed in Alzheimer's disease. In order to investigate the distribution of lesions associated with aging as well as with the earliest symptoms of senile dementia, we performed a quantitative neuropathologic evaluation of a large series of elderly patients representing the entire autopsy population for the year 1989 from a geriatric hospital. Among the 145 cases quantitatively assessed, there were 102 nondemented patients, 33 patients presenting clinically with globally intact intellectual function but early signs of impairment of specific cognitive functions, and 10 cases with senile dementia of the Alzheimer type. All of the cases had NFTs in layer II of the entorhinal cortex, regardless of their clinical diagnosis, and most cases had some NFTs in the CA1 field of the hippocampus. Severe pathologic changes within the inferior temporal neocortex were observed only in the demented cases. The extent of amyloid deposition was not correlated with the clinical diagnosis and seemed to be present in the neocortical areas earlier than in the hippocampal formation. Also, several cases contained NFTs without amploid deposition, but amyloid never occurred without NFTs. These results suggest that involvement of certain structures within the hippocampal formation is a consistent feature of aging. Thus, involvement of the hippocampal formation may be a necessary, but not sufficient, condition for the clinical expression of dementia, which is likely to be more closely related to the progressive degeneration of select neuronal populations in the neocortex.
Constantin Bouras, ${ }^{1.2}$ Patrick R. Hof, ${ }^{2,3}$ Pantèleimon Giannakopoulos, ${ }^{+}$Jean-Pierre Michel, ${ }^{+}$and John $\mathrm{H}$. Morrison ${ }^{2.3}$

'Department of Psychiatry, IUPG Bel-Air, University of Geneva School of Medicine, 1225 Geneva, Switzerland, 'Fishberg Research Center for Neurobiology and ${ }^{3}$ Department of Geriatrics and Adult Development, Mount Sinai School of Medicine, New York, New York 10029, and ${ }^{4}$ Geriatric Hospital, University of Geneva School of Medicine, 1226 Geneva, Switzerland

Neurofibrillary tangles (NFTs), senile plaques (SPs), and loss of large neurons are three pathological findings commonly observed in the cerebral cortex of Alzheimer's disease cases (Tomlinson et al., 1970; Ball, 1977; Terry et al., 1981; Mountjoy et al., 1983). The laminar and regional localization pattern of NFTs and SPS within the cerebral cortex indicates that a selective degeneration of long corticocortical and hippocampal projection systems is likely to occur (Mutrux, 1947; Pearson et al., 1985; Rogers and Morrison, 1985; Hyman et al., 1986, 1988, 1990; Lewis et al., 1987; Hof and Morrison, 1990; Hof et al., 1990a,b; Arnold et al., 1991; Braak and Braak, 1991; De Lacoste and White, 1993), leading to a disconnection of the cerebral cortex and resulting in the disintegration of intellectual functions observed in Alzheimer's disease patients (Pearson et al., 1985; Rogers and Morrison, 1985; Hyman et al., 1986; Lewis et al., 1987; Hof and Morrison, 1990; Hof et al., 1990a,b). In fact, Hyman and colleagues have demonstrated that the presence of NFTs in layer II of the entorhinal cortex was correlated by the presence of pathologic profiles in the termination zone of the perforant pathway in the outer portion of the molecular layer of the dentate gyrus, implying that this cortical pathway is severely affected in Alzheimer's disease cases (Hyman et al., 1988). In addition, the distribution of NFTs and SPS in the neocortex and in the hippocampal formation suggests that SP formation may involve the terminal arborization of NFT-bearing neurons (Pearson et al., 1985; Rogers and Morrison, 1985; Lewis et al., 1987; Hof and Morrison, 1990; Hof et al., 1990a; Senut et al., 1991; De Lacoste and White, 1993).

The distribution and density of these lesions in the cerebral cortex have been correlated with the degree of cognitive impairment in Alzheimer's disease. For example, Tomlinson et al. (1970) have reported a significant correlation between the mean SP frequency from several areas of the cerebral cortex and dementia severity assessed within 6 weeks of death using the Blessed Dementia Scale. However, these authors did not document the relationship between cortical NFT formation and dementia severity in their series. Other quantitative evaluations of the association of SPs and NFTs with dementia severity in Alzheimer's disease demonstrated a substantially greater correlation of cortical NFT than SP density, particularly in the temporal neocortex and parahippocampal gyrus (Wilcock and Esiri, 1982; Arriagada 
et al., 1992a). In addition, the severity of dementia has been shown to be highly correlated with synapse loss in neocortical areas (Terry et al., 1991).

The presence of Alzheimer's disease-related lesions in the brains of nondemented elderly individuals is well established (Tomlinson et al., 1968; Dayan, 1970; Ball, 1977; Mountjoy et al., 1983; Ulrich, 1985). More recently, several studies have demonstrated that amyloid deposition and NFT formation not only are common features of brain aging, but are found in certain regions of the cerebral cortex in densities that would qualify for a neuropathologic diagnosis of Alzheimer's disease cases (Crystal et al., 1988; Katzman et al., 1988; Hubbard et al., 1990; Morris et al., 1991; Price et al., 1991; Arriagada et al., 1992a,b; Hof et al., 1992; Berg et al., 1993; Bouras et al., 1993). In particular, a careful analysis of such cases revealed that in this population, the hippocampal formation systematically displayed a moderate to severe involvement, whereas the neocortical areas were unaffected or showed very low densities of NFTs and SPs (Price et al., 1991; Arriagada et al., 1992a; Hof et al., 1992; Berg et al., 1993; Bouras et al., 1993). This difference in regional lesion distribution distinguishes these elderly nondemented cases from the Alzheimer's disease population, in which neocortical areas are devastated (Pearson et al., 1985; Lewis et al., 1987; Hof et al., 1990a,b, 1992; Bouras et al., 1992). Most of the recent clinicoanatomic analyses of brain aging have been based on relatively small patient samples consisting of selected cases (Crystal et al., 1988; Katzman et al., 1988; Hubbard et al., 1990; Morris et al., 1991; Price et al., 1991; Arriagada et al., 1992a,b; Hof et al., 1992). In the present study, we had the opportunity to survey all of the autopsied cases from a geriatric hospital for the year 1989. We thus analyzed a large number of nonselected cases from a nonpsychiatric hospital in order to assess the degree to which elderly patients are affected by Alzheimer's disease-related neuropathologic changes, and to evaluate the age. related factors leading to NFT formation and amyloid deposition in a nondemented population. Part of this study has been reported in abstract form (Bouras et al., 1992).

\section{Materials and Methods}

\section{Survey of Population}

We surveyed the entire population of autopsy cases from the Geriatric Hospital of the University of Ge. neva School of Medicine (Switzerland) for the year 1989. This hospital is a large university-based institution ( 256 beds) that functions as the main geriatric care center for the greater Geneva area (about 400,000 inhabitants). A total number of 176 cases, all older than 65 years of age, were included in the present study (Table 1$)$. There were 77 men $(81.2 \pm 7.5$ years old; age range, $65-95 ; 43.75 \%$ ) and 99 women $(84.7$ \pm 7.5 years old; age range, $66-101 ; 56.25 \%$ ). Clinical data on the patients were obtained from the medical records of the Geriatric Hospital and from the Neu- ropathological Database of the Division of Morphological Psychopathology, Department of Psychiatry, University of Geneva School of Medicine, Geneva, Switzerland. All cases were neuropsychologically evaluated using a Mini-Mental State Examination (Folstein et al., 1975). Although the vast majority of the patients were hospitalized for terminal illness, all had been tested neuropsychologically at least 4 months prior to death. However, in some demented cases presenting with terminal somatic illnesses (i.e., metastatic tumor, severe cardiac and/or renal insufficien. cy) as well as in a few cases with Wernicke-Korsakoff encephalopathy, neuropsychological evaluation was no longer tenable. These cases (orher dementia cases, $n=28$ ) were subsequently excluded from the anatomoclinical correlations, although they were used in the general neuropathological evaluation. In addi. tion, three cases were also excluded from the analysis because it was impossible to assess their mental and cognitive function at admission (one oligophrenic man, 85 years old, and two women presenting with severe aphasia due to extensive vascular lesions, 87 and 89 years old, respectively). Thus, for anatomoclinical correlations, 145 cases (60 men, $79.6 \pm 8.2$ years old, age range $65-95$, and 85 women, $83.3 \pm$ 7.5 years old, age range 66-101) were considered and were subdivided into three diagnosis groups accord. ing to clinical and neuropathological findings (Table 1 ). Nondemented patients (ND cases, $n=102$ ) pre sented with preserved intellectual functions and showed no sign of temporospatial disorientation and memory impairment. Demented patients exhibited a greater variability in the clinical presentation. For instance, some cases presented with severe disorientation and memory impairment (DMI cases, $n=33$ ), but without aphasia-apraxia-agnosia syndrome. Other cases exhibited clinically the typical symptomatology of degenerative dementia of the Alzheimer type (AD cases, $n=10$ ), with marked temporospatial disorientation and cognitive impairment, aphasia, apraxia and agnosia, and severe handicap in daily living. Alzheimer's disease was subsequently confirmed neuropathologically in all cases from this group. Finally, a large number of cases of all three diagnosis group displayed variable degrees of vascular damage $(n=$ 107). This finding is not uncommon in the brain of elderly patients, and a recent neuropathologic study of 50 demented cases has revealed that up to $30 \%$ of the cases suffered from mixed dementia (Fallet-Bianco et al., 1990).

A full routine neuropathological evaluation of all the cases was performed on selected cortical areas (superior frontal, inferior temporal, inferior parietal, primary and secondary visual cortex, and anterior hippocampal formation). The densities and severity of neuropathological changes (i.e., NFTs, amyloid deposition, gliosis, microinfarcts, cortical atrophy) were assessed for each area separately and filed in a custom database. The total number of NFTs and amyloid-positive profiles was assessed from a series of random 1 $\mathrm{mm}^{2}$ cortical samples in all of the cortical areas surveyed, for each case separately. For this purpose, am- 


\begin{tabular}{lllll}
\hline $\begin{array}{l}\text { Table 1 } \\
\text { Distribution of the diagnosis groups }\end{array}$ & & & \\
& & & \\
Diag- & Age & Sex & Fre- & \\
nosis & $( \pm S D)$ & (M/N) & quency & $\%$ \\
\hline ND & $81.3 \pm 7.8$ & $43 / 59$ & 102 & 59.0 \\
DMl & $83.1 \pm 7.4$ & $17 / 16$ & 33 & 19.0 \\
AD & $88.6 \pm 4.8$ & $0 / 10$ & 10 & 5.8 \\
Other & $87.8 \pm 5.1$ & $16 / 12$ & 28 & 16.2 \\
\hline
\end{tabular}

$\mathrm{ND}$, nondemented; $\mathrm{DMI}$, disorientation and memory impairment; $\mathrm{AD}$, degenerative dementia of the Alzheimer type; Other, other dementia (sefers to demented cases of various etiology). A total number of 145 patients were considered. Due to incomplete clinical data, a total of 31 cases were excluded from the analysis (the other dementia group and three additional cases; see Materials and Methods). MN. men/women.

yloid-positive profiles were not differentiated into morphologic subgroups, and diffuse deposits, classical and core plaques were considered together for the assessment of amyloid deposition within the selected cortical regions. Depending on the density of lesions, cases were subsequently subdivided for neuroparhologic assessment into four groups displaying an absence of lesions, rare lesions ( $<10$ NFTs or amyloid-positive profiles per $\mathrm{mm}^{2}$ ), moderate densities of lesions (10-20 NFTs or amyloid-positive profiles per $\mathrm{mm}^{2}$ ), or frequent lesions ( $>20$ NFTs or amyloidpositive profiles per $\mathrm{mm}^{2}$ ), respectively. All of the cases with a clinical history of dementia were rated as having frequent lesions in all of the cortical areas surveyed, with lesion densities compatible with those recommended by the CERAD guidelines (Mirra et al., 1991, 1993). Additional tissues were available for de tailed analysis and immunohistochemistry.

\section{Tissue Collection and Staining Procedure}

All the brains were collected at autopsy (postmortem delay, 2-24 hr), fixed, and subsequently stored in a large volume of $10 \%$ formalin solution. For routine neuropathological evaluation $20-\mu \mathrm{m}$-thick section were prepared from the selected areas (see above) and were stained with modified Globus (Globus, 1927; Hof et al., 1990a) and Gallyas (Gallyas, 1971; Hof et al, 1990a) silver impregnation techniques, Luxol-fast blue-Van Gieson, cresyl violet, and hematoxylin-eo. sin. In addition, a modified thioflavine $S$ method was used for routine semiquantitative neuropathologic assessment (Guntern et al., 1992). This method yields results comparable to those obtained on tissues stained with antibodies to the microtubule-associated protein tau or to the amyloid $\beta$ A4 protein (Vallet et al., 1992). Briefly, 20- $\mu \mathrm{m}$-thick sections were mounted onto poly$L-$ lysine-coated slides. Then, they were treated with

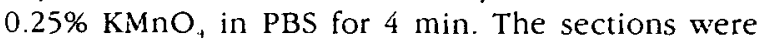
then placed in a solution consisting of $1 \% \mathrm{~K}_{2} \mathrm{~S}_{2} \mathrm{O}_{4}$ and $1 \%$ oxalic acid in PBS for $2-3 \mathrm{~min}$, rinsed, and stained with $0.0125 \%$ thioflavine $S$ in $40: 60100 \%$ ethanol:PBS (v/v ratio) for $3 \mathrm{~min}$. They were finally differentiated in 50\% ethanol in PBS and coverslipped with glycerin: $\mathrm{H}_{2} \mathrm{O}$ (3:1). With this method, lesions can be easily identified under fluorescence lighting conditions us. ing a Zeiss narrow-bandpass fluorescein filter set. For immunohistochemistry, additional sections were pro- cessed with a specific antibodies to the microtubuleassociated protein tau and to the amyloid $\beta$ A 4 protein. Characterization and specificity of these antibodies have been fully reported elsewhere (Défossez et al., 1988; Kim et al., 1988; Delacourte et al., 1990). Briefly, $20-\mu \mathrm{m}$-thick sections were incubated overnight with either the anti-tau antibody or the anti- $\beta$ A4 antibody, both at a dilution of 1:4000. Following incubation, sections were processed by the PAP method using diaminobenzidine as a chromogen.

\section{Quantitative Analysis}

NFTs and SPS were counted on a series of nine sections stained with thioflavine $S$ (sections $1,4,7$ ), antitau antibody (sections $2,5,8$ ), and anti- $\beta$ A 4 antibody (sections $3,6,9$ ). In the present study, quantitative analysis was performed only on sections from the anterior hippocampus, entorhinal, rostral half of inferior temporal cortex (Brodmann area 20), superior frontal cortex (Brodmann area 9), and primary and secondary visual cortex (Brodmann areas 17 and 18). For quantitative purposes, only classical and core plaques were counted and referred to as SPS. Lesion densities were assessed by two independent investigators (C.B., P.R.H.), with very high interrater reliability ( $>95 \%$ ). In all of the cases, NFT and SP densities were cal. culated per $\mathrm{mm}^{2}$ within each cortical layer for each selected area (five laminar measurements per sample). All quantitative analyses were performed on a computer-assisted microscopy system consisting of a Zeiss Axioplan photomicroscope equipped with a motorized stage, high-sensitivity video camera ( $\mathrm{LH}$ 4036 LHESA Electronics), a Compaq Deskpro 386/20 microcomputer, and an SAMBA 2005 software devel. oped by TITN Inc. (Alcatel; Grenoble, France). Statistical analysis was performed using analysis of variance to compare distribution of lesions (NFTs, SPS) in the different diagnosis groups. Correlations be tween presence of lesions, age, and severity of the disease were calculated for the different cortical regions.

\section{Results}

\section{Regional Patterns of NFT and SP Distribution}

In all of the 10 cases presenting with a clinical symp. tomatology of dementia and with neuropathologically confirmed Alzheimer's disease, NFT and SP distributions were comparable to previous descriptions in all the areas investigated (Pearson et al., 1985; Rogers and Morrison, 1985; Lewis et al., 1987; Hof et al., 1990a; Arnold et al., 1991; Braak and Braak, 1991). For instance, NFTs were widespread in the hippo. campal formation and predominated in the CA1 field, subiculum, and layers $I I$ and $V$ of the entorhinal cortex. In the neocortex, NFTs were present in both layers III and V (Figs. 1, 2B,D), and were more numerous in the inferior temporal cortex than in the superior frontal, inferior parietal, and occipital cortex. SPs were present in high densities in all cortical areas and were preferentially located in the supragranular layers within the neocortical regions. Furthermore, there 

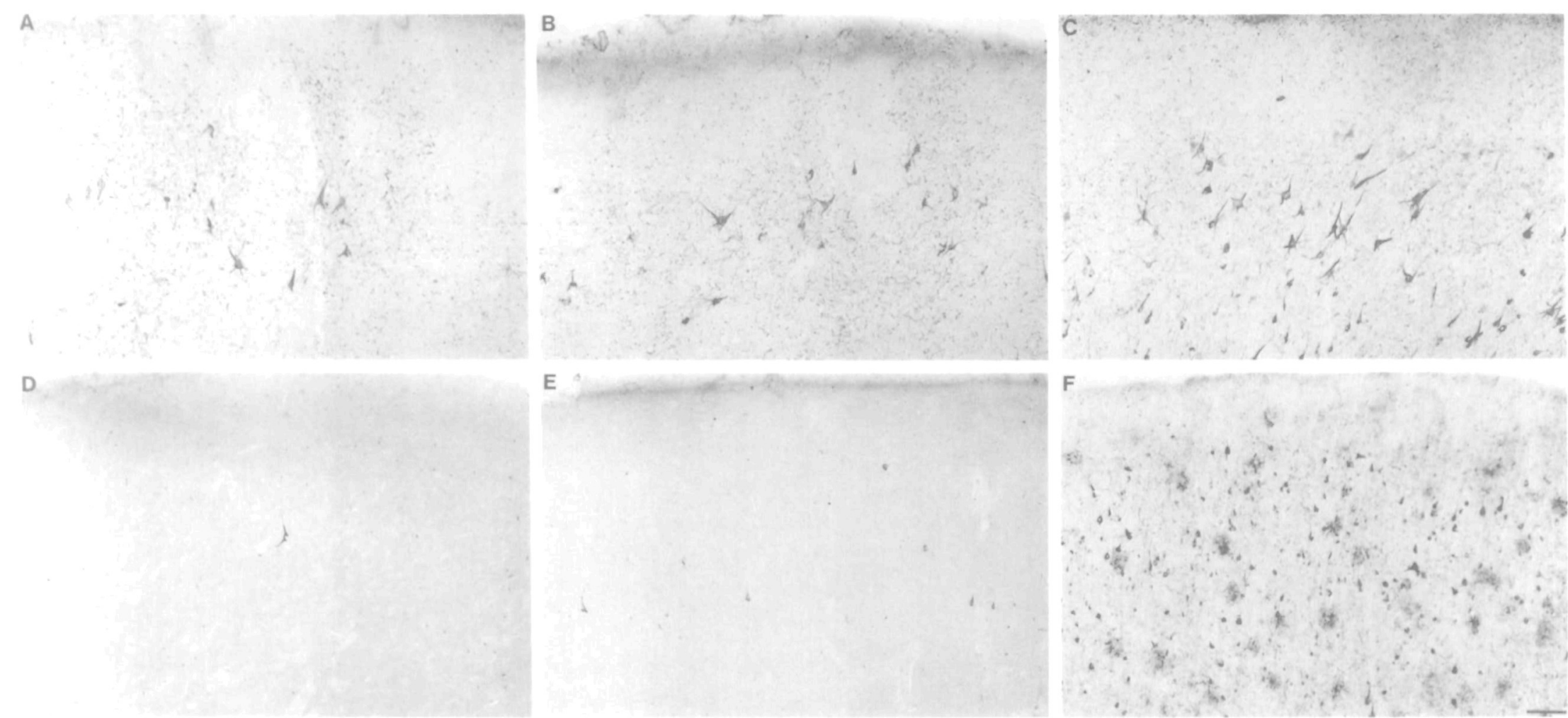

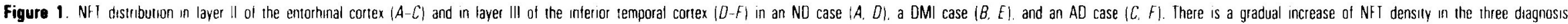

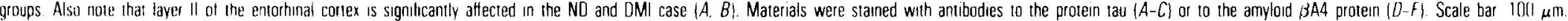



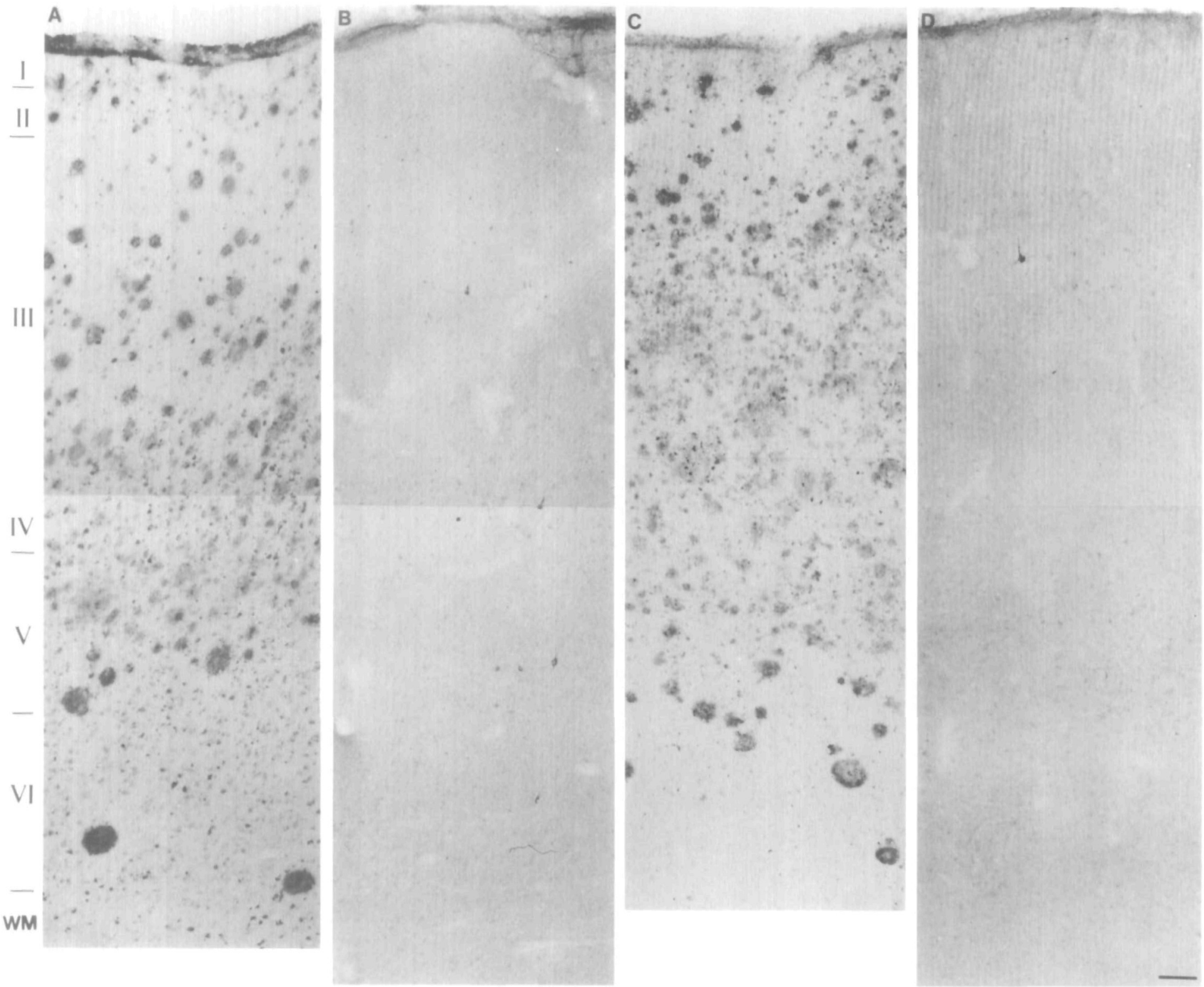

tion of SPS $(A, C)$ and NFTS $(B, D)$ in the inferior temporal cortex $(A, B)$ and superior frontal cortex $(C, D)$ in an ND case. Note the presence of very rare NFTs in both,

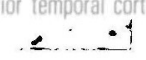
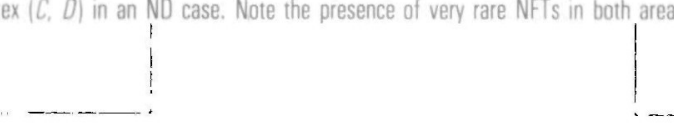

Figure 2. Distribution of $S P S(A, C)$ and NFTs $(B, D)$ in the inferior temporal cortex $(A, B)$ and superior frontal cortex $(C, D)$ in an ND case. Note the presence of very rare NFTs in both areas $(B, D)$, contrasting with the high density of $S P S(A, C)$. Materials were stained with antibodies to the amyloid $\beta A 4$ protein $(A, C)$ or the protein lau $(B, D)$. Cortical layers are indicated by Roman numerals; WM. white matter. Scale bar, $100 \mu \mathrm{m}$. 
Table 2

Incidence of lesions in the different diagnosis groups

\begin{tabular}{|c|c|c|c|c|}
\hline & ND & DMI & $A D$ & Other \\
\hline \multicolumn{5}{|l|}{ NFT } \\
\hline $\begin{array}{l}\text { Absent } \\
\text { Present }\end{array}$ & $\begin{array}{c}0(0) \\
102(100)\end{array}$ & $\begin{array}{c}0(0) \\
33(100)\end{array}$ & $\begin{array}{c}0(0) \\
10(100)\end{array}$ & $\begin{array}{c}0(0) \\
28(100)\end{array}$ \\
\hline \multicolumn{5}{|l|}{$S P$} \\
\hline $\begin{array}{l}\text { Absent } \\
\text { Present }\end{array}$ & $\begin{array}{l}59(57.8) \\
43(42.2)\end{array}$ & $\begin{array}{l}11(34.4) \\
21(65.6)\end{array}$ & $\begin{array}{l}1(10.0) \\
9(90.0)\end{array}$ & $\begin{array}{r}7(25.0) \\
21(75.0)\end{array}$ \\
\hline
\end{tabular}

Results represent the total number of patients ( $n=176$ ) with presence or absence of NFTs, SPS, and vascular involvement in each diagnosis group. Percentages of cases within each diagnosis group are indicated in parentheses.

was no noticeable difference in SP distribution and density berween the inferior temporal and superior frontal cortex (Fig. 2A,C). The visual areas of the occipital cortex had relatively high SP densities predominating in layer III and IV of the primary visual cortex and in layer III of the secondary visual cortex. Only rare NFTs were encountered in layer III of the secondary visual cortex in the AD cases. Also, the presence of NFTs and SPs was not related to the occurrence of alcoholism, smoking, or intercurrent somatic disorders in our population. Men were affected as frequently as women, with the exception of the AD group, which comprised only women, although this difference may be due to the small size of this particular diagnosis category $(n=10)$.

In the cases presenting with disorientation and mild cognitive impairment (DMI) as well as in the nondemented cases (ND), the general distribution of NFTs and SPs was qualitatively similar to that observed in the demented (AD) cases; however, large differences in lesion density were seen in all cortical regions in both groups. In all these cases, NFTs were systematically present in layer II of the entorhinal cortex (Fig. $1 A-C$, Table 2 ). In this respect, it is worth noting that in some cases of the ND group, NFTs were observed exclusively in this layer and that the re. mainder of the hippocampal and neocortical fields were devoid of NFTs. In the CA1 field and in the subiculum, NFTs were observed in variable densities in the ND and DMI groups. In both ND and DMI cases, NFTs were rare in the neocortical areas and, when present, were preferentially distributed in layer III of the inferior temporal cortex (Fig. $1 D-F$ ). Very low numbers of NFTs were encountered in the superior frontal cortex of these cases. It should be noted that the inferior parietal cortex also contained very low NFT densities in these cases, although no quantitative assessment was made in this region. In both groups, SPs were present in highly variable densities in all cortical regions (Fig. $2 A, C$ ), and were more frequently observed in the inferior temporal cortex. Numerous cases $(57.8 \%$ of the ND cases, $34.4 \%$ of the DMI cases, and $10.0 \%$ of the AD cases; Table 2 ) were devoid of amyloid deposition. It should also be noted that in some cases with NFT restricted to the entor. hinal cortex, a high density of amyloid deposition in the neocortex was observed in absence of clinical symptomatology (Fig. $2 A, C$ ). In addition, there was no difference in lesion densities between men and women, and there was a correlation between age and the presence of NFT ( $r=0.70, p<0.005)$, whereas such a correlation was not observed for SP (Table 3 ).

\section{Quantitative Analysis}

A laminar and regional quantitative assessment of the number of NFT in the different regions of the hippocampal formation and neocortex revealed striking differences between the diagnosis groups. In the hippocampal formation, the entorhinal cortex had the most severe involvement in all three diagnosis groups. The ND group had NFT densities of $7.4 \pm 0.8$ per $\mathrm{mm}^{\prime}$ in layer II, and $5.4 \pm 1.2$ in layer $V$. These values were $21.0 \pm 2.5$ and $6.9 \pm 3.2$ in the DMI group, and $59.1 \pm 12.4$ and $31.7 \pm 5.8$ in the AD group, respec. tively. The CAl field of the hippocampus showed slightly lower NFT densities as compared to the entorhinal cortex with values of $5.3 \pm 1.2$ (ND), $13.3 \pm$ 2.1 (DMI), and $87.5 \pm 20.1$ (AD) NFTs per $\mathrm{mm}^{2}$. The subiculum had NFT counts comparable to those observed in the CA1 $(6.9 \pm 1.3$ in the ND cases, $11.7 \pm$ 2.2 in the DMI cases, and $38.7 \pm 8.7$ in the AD cases). In the neocortex, most of the NFT were localized in the temporal areas and tended to be concentrated in layer III (Figs. 1,2). In all our cases, the frontal cortex

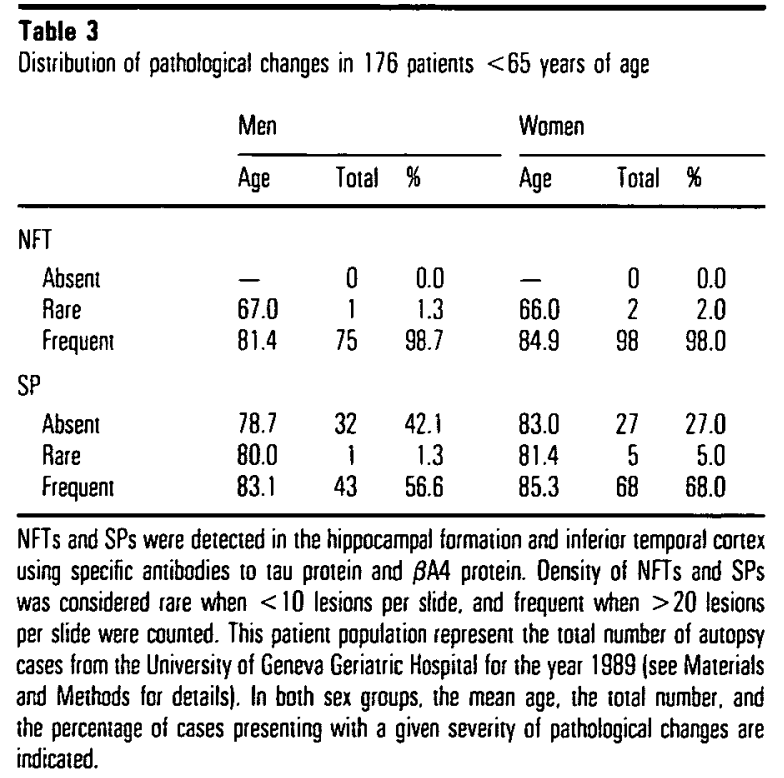


contained fewer NFT than the temporal regions. Thus, the inferior temporal cortex had NFT densities of 6.2 \pm 1.2 in layer III and $4.3 \pm 0.8$ in layer V (ND cases), $12.1 \pm 1.9$ and $7.8 \pm 1.5$ (DMI cases), and $46.9 \pm 6.5$ and $25.3 \pm 7.8$ (AD cases). In the superior frontal cortex these combined values for layers III and $V$ were $4.9 \pm 0.5,5.6 \pm 1.0$, and $7.7 \pm 3.3$ in the ND, DMI, and $A D$ groups, respectively. Finally, NFT densities in layer II of the entorhinal cortex and CA1 were statistically correlated with the presence of disorientation and mild cognitive impairment $(p<0.001)$, and Alzheimer's disease was further correlated with the involvement of layer III of the inferior temporal cortex $(p<0.005)$.

SPs were observed in all three diagnosis groups and were more numerous in the demented patients The ND cases displayed generally fewer SP than the cases from the two other groups. In addition, the neocortex contained more SPs than the hippocampal formation in all the cases. For instance, the inferior tem. poral correx had SP densities of $6.1 \pm 0.9,14.6 \pm 2.1$, and $13.9 \pm 3.4$ per $\mathrm{mm}^{2}$ in layer III of the ND, DMI and $\mathrm{AD}$ cases, respectively, and $4.4 \pm 0.7,10.5 \pm 1.6$, and $7.9 \pm 2.5$, respectively, in layer $V$. The superior frontal cortex showed SP counts of $4.7 \pm 0.6,5.8 \pm$ 0.9 , and $12.9 \pm 3.3$ in layers III and $V$ of the three groups, respectively. These values were $8.6 \pm 2.7,7.8$ \pm 1.1 , and $19.6 \pm 7.1$, respectively, in layers III and IV of the primary visual cortex, and $9.7 \pm 2.9,7.2 \pm$ 0.9 , and $17.6 \pm 6.5$, respectively, in layers III and V of the secondary visual cortex. In the hippocampal formation, the CA1 field contained the highest SP densities, with values reaching $0.2 \pm 0.1$ in the ND cases, $5.7 \pm 1.1$ in the DMI cases, and $10.0 \pm 1.0$ in the AD cases. The subiculum contained $0.2 \pm 0.1,3.2$ \pm 1.8 , and $3.8 \pm 0.8 \mathrm{SPs}$ per $\mathrm{mm}^{2}$, respectively. In the entorhinal cortex, the superficial layers displayed SP densities of $0.6 \pm 0.3,7.2 \pm 1.4$, and $8.0 \pm 1.7$, respectively, while in the deep layers, these values were $0.6 \pm 0.3,5.3 \pm 1.1$, and $6.5 \pm 0.6$. Thus, the presence of amyloid deposition was related to the clinical di. agnosis of dementia in neocortical regions; however, no difference could be found between the DMI and AD groups. Also, it should be mentioned that despite the relationship between the occurrence of amyloid and dementia, there were cases devoid of amyloid deposition in all three patient groups, as well as ND cases displaying high numbers of SPS in the neocortex.

\section{Relationsbip between Presence of NFTs and SPs} It should be mentioned that although NFTs were al. ways visualized within layer II of the entorhinal cortex in all cases, they were not necessarily present in all cortical regions in any given case. For instance, depending on the cortical area, up to $87 \%$ of the cases presented without NFTs and $81 \%$ without SPs in at least one cortical region in the ND group, while these values were $79 \%$ and $51 \%$, respectively, in the DMI cases, and $70 \%$ and $30 \%$ in the AD cases (Table 4 ). Also, it is worth noting that some ND cases with NFTs mostly restricted to laver II of the entorhinal cortex and CA1 field contained large amounts of amyloid deposition throughout the cerebral cortex (Fig. $2 \mathrm{~A}$ $D)$. However, no case had amyloid deposition without NFTs, except in the visual areas of the occipital cortex, which were basically devoid of NFTs and had a relatively high density of SPs in all of the cases (Table 4). Moreover, several cases $(31.4 \%$ of ND cases, $12.5 \%$ of DMI cases, no AD cases) presented with moderate-to-high NFT densities but were devoid of amyloid deposition. A regional analysis of the coex istence of NFT and amyloid deposition (including diffuse preamyloid deposits, classical and core plaques) revealed that $26.4 \%$ of all cases had NFT in layer III of the inferior temporal cortex without amyloid deposition in this region. Similarly, $59.0 \%$ of all cases had NFTs in layer II of the entorhinal cortex, and $39.8 \%$ in the CA1 field of the hippocampus in absence of colocalized amyloid deposition. In addi tion, $45.0 \%$ of the cases displayed NFTs in the entorhinal cortex in absence of amyloid in the temporal cortex, whereas $36.6 \%$ had NFTs in the CA1 and no amyloid in the entorhinal cortex. Finally, $68.0 \%$ of the cases had NFTs in layer II of the entorhinal cortex and no amyloid in the CA1 field, and $25.3 \%$ showed NFTs in the inferior temporal cortex and no amyloid in the superior frontal cortex. These observations indicate that there is a minimal correlation between the presence of these two lesions in anatomically related areas. However, there was a clear correlation between NFT counts in regions or cortical layers that are anatomically linked. Thus, a strong correlation was observed berween NFT densities in layer II of the entorhinal cortex and in the CAl field $(r=0.87, p<$ $0.001)$, in the subiculum and layer $V$ of the entorhinal cortex $(r=0.75, p<0.001)$, and in layer $\mathrm{V}$ of the entorhinal cortex and layer III of the inferior temporal cortex $(r=0.84, p<0.001)$.

\section{Discussion}

The present neuropathologic analysis of 176 elderly individuals reveals that many features observed in the brains of patients presenting with Alzheimer's disease can also be found in a geriatric population, although the severity of the changes is not as dramatic as in Alzheimer's disease, and the distribution of pathologic profile is quite different (Bouras et al., 1992). In particular, it is relevant to note that all of the cases in our study demonstrated NFTs in layer II of the entorhinal cortex, although 102 of these cases showed no signs of cognitive and memory impairment at death. Similarly, $57 \%$ of the ND cases had a few NFTs in the CA1 field of the hippocampus, and $78 \%$ in the inferior temporal cortex. SPs were not always observed and although they tended to be more numerous in the demented cases, there were clinically demented cases containing only a few SPs. However, the NFT distribution pattern suggests that the entorhinal cortex is affected first, followed by the CAl field, the subiculum, the inferior temporal cortex, and finally other neocortical regions. Conversely, as recently pointed out by Arriagada and colleagues, we observed that amyloid deposition appears to occur first in neocortex 
rather than in the hippocampal formation (Arriagada et al., 1992b; Bouras et al., 1992), regardless of the diagnosis group. However, when present in the neocortex of ND cases, SP counts were within the range of SP densities observed in the AD cases. Also, the superior frontal cortex displayed relatively low densities of NFTs and SPs and was involved more severely only in the AD group.

These data suggest that some of the DMI and ND cases may represent very early stages of Alzheimer's disease, in that the inferior temporal correx in these cases might represent an interface between substantially affected and unaffected areas, indicating that this area may be a reliable landmark to study the relationships between the clinical observations and pathologic findings. In particular, it emerges from our analysis that even moderate-to-severe involvement of the entorhinal cortex and CA1 field of the hippocampus can be considered compatible with an apparently normal cognitive status or a mild cognitive impairment and disorientation that still allows for indepen. dent daily living. However, once the degenerative changes involve the neocortex, clinical signs of dementia are observed, regardless of the age of the patients. This suggests that at all ages within a population of elderly individuals, the implication of neocortical areas is a necessary factor for the clinical expression of the dementia. Although the vast majority of our cases clearly were not demented, the slight cognitive disturbances noted in the DMI cases are similar to the early impairments seen in patients who may subsequently progress to the global cognitive and behavioral disturbances that characterize Alzheimer's disease. Thus, the subtle cognitive disturbances evidenced in some ND and DMI cases are associated with a pattern of lesion distribution in the hippocam. pal formation that is qualitatively compatible with a neuropathological diagnosis of Alzheimer's disease. However, the neuropathological involvement of the neocortex differed dramatically from that of Alzheimer's disease patients, given that there were few NFTs outside of the hippocampal formation. This suggests that extensive cortical SP and NFT formation restricted to medial temporal areas may take place long before the emergence of overt clinical symptoms of dementia. Moreover, the relative preservation of global cognitive functioning in the ND and DMI patients might be related to the absence of widespread neocortical NFT formation. In this context, it is worth noting that in experimental models of memory impairment, macaque monkeys with lesions restricted to the hippocampus and amygdala displayed a sparing of their learning skills, whereas lesions of the perirhinal and parahippocampal fields TF and TH pro. duced severe memory disturbances, stressing the fact that these cortical fields are key elements of the mem. ory system (Zola-Morgan and Squire, 1984; Zola-Morgan et al., 1989; Squire and Zola-Morgan, 1991; Suzuki et al., 1993).

The present results parallel previous observation of Alzheimer's disease-related pathologic changes in the brain of old patients with no signs of neurologic

\begin{tabular}{|c|c|c|c|}
\hline & ND & DMI & $A D$ \\
\hline \multicolumn{4}{|l|}{ CA1 } \\
\hline $\begin{array}{l}\text { NFT } \\
\text { SP }\end{array}$ & $\begin{array}{l}57 \\
19\end{array}$ & $\begin{array}{l}73 \\
49\end{array}$ & $\begin{array}{r}100 \\
70\end{array}$ \\
\hline \multicolumn{4}{|c|}{ Subiculum } \\
\hline $\begin{array}{l}\mathrm{NFT} \\
\mathrm{SP}\end{array}$ & $\begin{array}{l}56 \\
28\end{array}$ & $\begin{array}{l}64 \\
49\end{array}$ & $\begin{array}{r}100 \\
70\end{array}$ \\
\hline \multicolumn{4}{|c|}{ Entorhinal cortex } \\
\hline $\begin{array}{l}\text { NFT } \\
\text { SP }\end{array}$ & $\begin{array}{r}100 \\
30\end{array}$ & $\begin{array}{r}100 \\
50\end{array}$ & $\begin{array}{r}100 \\
70\end{array}$ \\
\hline \multicolumn{4}{|c|}{ Inferior temporal cortex } \\
\hline $\begin{array}{l}\text { NFT } \\
\text { SP }\end{array}$ & $\begin{array}{l}78 \\
42\end{array}$ & $\begin{array}{l}84 \\
66\end{array}$ & $\begin{array}{l}90 \\
90\end{array}$ \\
\hline \multicolumn{4}{|c|}{ Superior frontal cortex } \\
\hline $\begin{array}{l}\text { NFT } \\
\text { SP }\end{array}$ & $\begin{array}{l}13 \\
44\end{array}$ & $\begin{array}{l}21 \\
61\end{array}$ & $\begin{array}{l}30 \\
90\end{array}$ \\
\hline \multicolumn{4}{|c|}{ Primary visual cortex } \\
\hline $\begin{array}{l}\text { NFT } \\
\text { SP }\end{array}$ & $\begin{array}{r}0 \\
83\end{array}$ & $\begin{array}{r}0 \\
100\end{array}$ & $\begin{array}{r}0 \\
100\end{array}$ \\
\hline \multicolumn{4}{|c|}{ Secondary visual cortex } \\
\hline $\begin{array}{l}\text { NFT } \\
\mathrm{SP}\end{array}$ & $\begin{array}{r}0 \\
75\end{array}$ & $\begin{array}{r}0 \\
100\end{array}$ & $\begin{array}{r}1 \\
100\end{array}$ \\
\hline
\end{tabular}

Results represent the percentages of cases in each diagnosis group presenting with NFTs and SPs. Note that layer II of the entorhinal contex is involved with NFT formation in $100 \%$ of the cases. These resulks reflect only the occursence of lesions in the three patient groups and therefore have a limited value in terms of correlation with severity of dementia (see Results for details).

or psychiatric disorders (Tomlinson et al., 1968; Da. yan, 1970; Terry et al., 1981; Mountjoy et al., 1983; Ulrich, 1985; Crystal et al., 1988; Katzman et al., 1988; Hubbard et al., 1990; Mann et al., 1990; Morris et al., 1991; Price et al., 1991; Arriagada et al., 1992a,b; Hof et al., 1992; Lippa et al., 1992; Vickers et al., 1992; Berg et al., 1993; Bouras et al., 1993). For example, Hubbard et al. (1990) reported a neuropathological survey of 70 unselected cases from a general hospital. In this series, four cases with documented evidence of cognitive impairment showed numerous NFTs in all neocortical areas examined, whereas six cases showing no signs of cognitive decline had excessive NFT formation confined to the hippocampal formation and temporal neocortex, but no NFTs in the other neocortical regions. These authors suggest that NFT formation confined to these areas may represent a preclinical stage of Alzheimer's disease. Similarly, the recent analysis of clinically assessed demented and nondemented cases by Berg et al. (1993) demonstrated that NFT formation was a strong correlate of duration and severity of dementia. In addition, this study shows that only neocortical, but not hippocampal, NFT densities represent a reliable index of dementia (Berg et al., 1993), suggesting that involvement of cortical structures outside of the hippocampal formation is a prerequisite for the development of de mentia. Also, it indicates that the dementing syndrome may progress in the neocortex in presence of relatively high and stable NFT densities in the hip- 
pocampus and entorhinal cortex (Berg et al., 1993; Bierer et al., 1993).

Furthermore, a retrospective neuropathological as. sessment of 12 cases aged over 100 years but without clinically documented evidence of cognitive impairment revealed dense NFTs in the CA1 field, moderate NFT involvement in temporal cortex, and absence of NFTs in other neocortical areas (Hauw et al., 1986). These elderly nondemented cases demonstrate NFT distribution quite similar to our ND cases, at least in the hippocampus proper. Results from our laboratory also demonstrate the preferential localization of NFT in the CA1 field of nondemented centenarians, sug. gesting that the distribution of lesions in these very old unaffected patients may be somewhat different as compared to nondemented younger cases, in that the entorhinal cortex is less involved in centenarians (Giannakopoulos et al., 1993). Thus, the distribution of neuropathologic changes in aging may be more complex than is usually thought, in that there may be subgroups of patients with specific neuropsychological profile that may correspond to the involvement of select regions, connections, and neuron popula. tions. Similar observations have been made by Braak and Braak (1990) in six parients with NFTs predom. inating in the entorhinal cortex, lower NFT counts in the CA1 and temporal neocortex, and numerous SPs in the temporal neocortex. These patients had a clinical diagnosis of probable senile dementia but insufficient cortical SP and NFT densities to meet neuro. pathologic criteria for the diagnosis of Alzheimer's disease. Although these authors did not report a quantitative analysis of their cases, the distribution pattern of the lesions is comparable to that observed in the present series.

Other recent studies have shown that the clinical dementia in Alzheimer's disease is associated with the presence of neocortical NFTs, but that SPs may be widespread in the cerebral cortex in the absence of symptoms of cognitive and memory decline. For instance, Katzman et al. (1988) described a series of cases clinically considered nondemented, with cortical SP distribution comparable to clinically and neuropathologically confirmed Alzheimer's disease cases, but with the absence of frontal and parietal NFT formation. NFT formation in the temporal neocortex and hippocampus of these cases was comparable to a control case series with minimal SP formation. Crystal et al. (1988) reported a small series of prospectively assessed elderly persons with cortical SP densities similar to Alzheimer's disease cases, but rare cortical NFTs, and ratings of cognitive function that did not warrant a diagnosis of dementia. These cases are also comparable to our ND and DMI cases since they showed mild to severe NFT densities and relatively low SP counts in the hippocampal formation. Four orher cases with anterogradely assessed Clinical Dementia Rating (CDR) scale scores of 0.5 (questionable dementia), with SP formation throughout the neocortex and NFTs confined to the hippocampal formation have recently been described (Morris et al., 1991). Similarly, we recently reported the case of a nondemented patient demonstrating globally intact intellectual function and initial symptoms of impaired cognitive functions 2 weeks before death (Hof et al., 1992). In this case, we found SP densities comparable to those obtained in Alzheimer's disease cases in neocortical areas, while high densities of NFTs were restricted to select areas of the medial and inferior temporal cortex, including hippocampus, entorhinal cortex, perirhinal and inferior temporal cortex (Hof et al., 1992). In a series of 25 patients prospectively or retrospectively evaluated for dementia, Price et al. (1991) demonstrated comparable densities and dis tribution of NFTs and SPS in 19 cases with CDR ratings of $0.5-1.0$, indicating minimal cognitive decline. Similarly, an analysis of the hippocampal formation and inferior temporal cortex in 61 nondemented elderly cases revealed that in each region the number of neurons affected by NFT formation, calculated from the total number of neurons, gradually increased with age and that eight of these cases consistently exhibited higher NFT counts in all of the areas investigated than the remainder of the patients (Bouras et al., 1993). In this series, no correlation between SP counts and NFT densities and age was observed (Bouras et al., 1993). Dickson et al. (1991) showed that cortical am yloid deposition can be used to subdivide two neuropathologic groups of elderly individuals character. ized by either minimal or severe amyloid deposition. However, these authors found no correlation between amyloid deposition and the cognitive performance of the patients (Dickson et al., 1991) and suggested that other factors such as cytoskeletal alterations are necessary for the development of dementia. All of these data suggest that widespread cortical amyloid deposition and NFT formation limited to certain hippocampal areas are not incompatible with intact cog. nitive abilities, yet extensive NFT formation in neocortex is clearly linked to cognitive decline.

The quantitative analysis of lesion distribution in the cerebral cortex of many elderly cases suggests that certain areas of the medial and inferior aspects of the temporal lobe are likely to represent an early site of NFT formation. In fact, NFT counts in the entorhinal cortex of such cases are elevated even in comparison to Alzheimer's disease cases with several years of evo. lution. In addition, biochemical studies have dem. onstrated the presence of abnormally phosphorylated forms of the tau protein in the entorhinal cortex of nondemented elderly persons, revealing the exis. tence in this particular area of a biochemical dys. function that is widespread in the cerebral cortex of Alzheimer's disease cases (Vermersch et al., 1992). The inferior temporal cortex, a visual association area, appears to be an early transition zone between the severely affected entorhinal and perirhinal cortex and the mid temporal gyrus in these cases (Hof et al., 1992). Thus, a progression in NFT density within adjacent cortical components of the medial and inferior aspects of the temporal cortex may be a neuropathological hallmark of incipient dementia in elderly patients presenting with normal cognitive abilities or very mild cognitive impairment. It should be kept in 
mind that, in absence of a extensive quantitative investigation of the cerebral cortex, it is still possible that there may be significant numbers of NFTs in cortical areas not examined in the present survey. In this context, the distribution of lesions in cortical regions related to the limbic system is of particular interest. A recent study has demonstrated that in Alzheimer's disease, NFTs are predominantly distributed within a restricted portion of the agranular orbitofrontal cortex (Chu et al., 1993). The involvement of such regions early in the progression of dementia could also account for some of the memory and behavioral disturbances presented by Alzheimer's disease patients. However, our comprehensive quantitative study of a nondemented individual with minimal signs of cognitive impairment revealed that the orbitofrontal, cingulate, as well as frontal, parietal, and mid and su. perior temporal cortex was essentially devoid of NFTs, suggesting that these areas may be affected at a later stage in the progression of the disease (Hof et al., 1992)

The fact that there is a strong correlation between NFT densities in anatomically related cortical regions is of special interest, since it has been proposed that Alzheimer's disease involves certain cortical circuits, in particular, systems related to memory within the hippocampal formation (Hyman et al., 1986, 1988, 1990; Senut et al., 1991), and corticocortical pathways in the neocortex (Pearson et al., 1985; Rogers and Morrison, 1985; Lewis et al., 1987; Hof and Morrison, 1990; Hof et al., 1990a,b; De Lacoste and White, 1993). Specifically, it has been suggested that the distribu. tion of NFTs and SPs is a reflection of the degeneration of certain corticocortical connections and that the dementia may progress along a defined set of projec. tions, eventually leading to a syndrome of cortical disconnection (Pearson et al., 1985; Rogers and Morrison, 1985; Lewis et al., 1987; Hof et al., 1990a,b). In fact, the laminar distribution of NFTs and SPs in areas participating in hierarchically organized distributed systems, such as the visual and auditory cortical pathways, reflects the origin and termination of corticocortical projections as defined in connectivity studies in nonhuman primate (Lewis et al., 1987; Hof et al., 1990a; De Lacoste and White, 1993). In this context, it is interesting that SP and NFT densities were not systematically correlated in our geriatric patient population, even in anatomically linked regions within the hippocampal formation. In fact, in agreement with other authors (Arriagada et al., 1992b), amyloid de. position seems to occur earlier in neocortical regions than in the hippocampal formation, and shows no correlation with the clinical diagnosis, or with NFT formation locally as well as in distant anatomically linked areas. This difference from previous observations on Alzheimer's disease cases may be due to the fact that the present set of cases comprises only 10 clinically documented dementia cases, and that the DMI and possibly some ND cases may represent early stages in the dynamic processes leading to the endstage cortical patterns of NFTs and amyloid deposition that are fully recognizable only in severe $\mathrm{Al}$ - zheimer's disease cases. Another pattern revealing putative dynamic pathologic events is that NFTs appear first in layer III of the inferior temporal cortex in our series, whereas in previous reports of severe Alzheimer's disease cases, they were distributed in both layers III and $\mathrm{V}$ with higher densities in layer $\mathrm{V}$ (Lewis et al., 1987; Hof et al., 1990b), suggesting that the supragranular layers might be involved in the degenerative process earlier than the infragranular lay. ers.

The analysis of this series of cases extends previous observations on aging and Alzheimer's disease in that it surveys a nonselected population of cases from a nonpsychiatric institution. Globally, the finding that all the cases display some degree of neuropatholog. ical involvement of the medial regions of the temporal lobe may be related to the fact that certain neurons in these areas are usually affected early during aging (Arriagada et al., 1992b; Vermersch et al., 1992; Vickers et al., 1992). In particular, the allocortex and paleocortical zones are damaged systematically in this population, regardless of the clinical neurologic and psychiatric status of the cases, whereas the neocortex appears to be severely involved only at a time when overt symptoms of dementia are present. In addition, these findings stress the problem of the reliability of neuropathologic rating scales for the diagnosis of Alzheimer's disease, and point to the fact that the presence of lesions in the hippocampal formation alone is clearly insufficient to establish the diagnosis of degenerative dementia, especially in patients presenting with mild cognitive impairment (Hof et al., 1992). For instance, the involvement of the entorhinal cortex and hippocampus proper in clinically nondemented patients could represent the neuropathologic basis for the age-associated memory impairment syndrome (otherwise called benign senescent forgetfulness; Crook et al., 1986; Gordon, 1992). These are middleaged as well as elderly patients presenting with noticeable forgetfulness, whereas their daily functioning and judgement capabilities are not impaired. Such correlations with early pathologic alterations and subtle changes in cognitive abilities should, however, been drawn with caution from the present data, since our patients were assessed with a relatively poorly sensitive instrument, although at least some of the ND and DMI cases in the present analysis could fit within this clinical definition. Interestingly, the NFT distribution in these cases corresponds precisely to the anatomic localization of neurons participating in memory-related circuits (Hyman et al., 1986, 1988, 1990; Vickers et al., 1992). However, the involvement of the hippocampal formation in the development of dementia may account for a small part of the symptomatology, but not for the massive intellectual decline and memory loss characteristic of patients with severe Alzheimer's disease, which is more likely to be correlated with the progressive damage of neocortical areas. In this context, Terry and colleagues have recently demonstrated a striking correlation be. tween the degree of dementia and the loss of synapses in several neocortical regions (Terry et al., 1991), 
indicating that dementia may be directly related to the loss of specific connections between association areas of the cerebral cortex.

The temporal separation of hippocampal and neocortical degeneration may have important implications for diagnosis of an early decline in mental status that may be a precursor to Alzheimer's disease, but is still compatible with independent daily living. The present data suggest that a significant proportion of the aged population may be functioning at a high level with substantial compromise of key hippocam. pal circuits. It is therefore possible that memory is highly dependent on hippocampal circuits at certain processing stages, but may become quite independent of the hippocampal formation, once addressing and retrieval of information is dependent on neocortical circuits (Hodges et al., 1992). In addition, the present results indicate that sensitive neuropsychological assessment of the aged population should be developed along two lines: first, memory assessment that is primarily or completely dependent on intact hippocampal circuits, and second, tests of visual func. tion directed at processes such as complex form analysis that are dependent on the inferior temporal gyrus Sensitive neuropsychological tests designed with these circuits in mind might detect deficits well in advance of the cognitive decline that is linked to further neocortical involvement. Should a mechanism become available that slows down or halts the degenerative process affecting corticocortical circuits beyond this relatively limited portion of the temporal lobe, de tection of the early degenerative events will be essential in order to initiate treatment while the capacity for independent living is still present.

Even though the distribution of NFT in aging ap pears to define select anatomic regions of the cerebral cortex, the issue that many cellular variables may be changing simultaneously in different cortical regions remains to be addressed. The analysis of cellular pathology in Alzheimer's disease revealed that only certain populations of neurons are prone to NFT for mation, and that specific morphologic and neurochemical characteristics of the affected cells are related to their heightened vulnerability. Recently, an association has been demonstrated between the high somatodendritic content in neurofilament protein of specific neuron subgroups and their involvement in NFT formation in the hippocampal formation and neocortex in intellectually normal elderly people (Vickers et al., 1992), as well as in Alzheimer's disease cases (Morrison et al., 1987; Hof and Morrison, 1990; Hof et al., 1990b). Conversely, other neuronal pop. ulations, such as morphologically distinct classes of interneurons that contain calcium-binding proteins, are unaffected even in severe cases of Alzheimer's disease (Hof and Morrison, 1991; Hof et al., 1991, 1993). This indicates that differential neuronal vulnerability in select elements of the cortical circuitry may be a crucial factor in both the neuropathologic changes observed in aging and in the progression of Alzheimer's disease. In particular, normal aging has been found associated with transitional cellular changes in layer II of the entorhinal cortex consisting of degenerating neurons labeled by antibodies to both neurofilament protein and abnormally phosphorylat. ed tau protein, whereas the prefrontal cortex in these cases showed no such profile (Vickers et al., 1992). Demented cases, however, contained end-stage NFTs in the entorhinal cortex, which had lost their antigenic characteristics, while transitional forms were present in the prefrontal cortex (Vickers et al., 1992). This analysis and previous results also indicated that NFTs are preferentially found by neurofilament pro. tein-containing neurons and that a severe loss of neurons without NFT formation is unlikely to occur, at least at early stages of the dementing process (Hof et al., 1990b; Vickers et al., 1992; Bouras et al., 1993).

These observations suggest that the cellular alterations that occur in Alzheimer's disease represent a dynamic and continuous process that affects different cortical fields at different points in time, the hippocampal formation being apparently involved earlier than the neocortex. Although the present results sug gest that the inferior temporal cortex may be the first neocortical field to be affected by these cellular changes, it has not yet been determined whether oth. er cortical regions are simultaneously involved. In addition, it should be mentioned that Alzheimer's disease is not the only form of dementia that severely affects the temporal cortex. For example, Pick's disease is also characterized by a massive involvement of the temporal lobe (Brion et al., 1991). However, a quantitative analysis of the neocortex in Pick's disease cases has revealed that the laminar distribution of Pick bodies involves small pyramidal neurons preferentially located in layers II and VI, which are usually not affected by NFT formation in Alzheimer's disease (Hof et al., 1994). This differential cellular distribution of lesions may account for some of the clinical differences observed between Pick's disease and Alzheimer's disease patients, as the neurons prone to Pick body formation clearly subserve different sets of cortical circuits. Thus, further studies of the molecular and morphologic phenotype of specific neuronal classes may allow for the definition of the key elements linked to their relative vulnerability in neurodegenerative disorders and may help in developing therapeutic strategies to protect the most sensitive cells from the pathologic process.

\section{Notes}

We thank M. Surini, P. Y. Vallon, and R. S. Woolley for expert technical assistance, Drs. A. Delacourte and N. K. Robakis for the generous provision of antibodies to tau and amyloid $\beta$ A4 proteins, and L. M. Bierer, E. A. Nimchinsky, and J. C. Vickers for critical reading of the manuscript. This study was supported by grants from the NIH (AG06647 and AG05138) the Brookdale Foundation, the Dana Foundation, and the American Health Assistance Foundation (to J.H.M. and P.R.H.).

Correspondence should be addressed to Dr. Patrick R Hof, Fishberg Research Center for Neurobiology, Box 1065 , Mount Sinai School of Medicine, One Gustave L. Levy Place, New York, NY 10029, or to Dr. Constantin Bouras, Department of Psvchiatr, ILPG Bel-Air, $\mathrm{CH}-1225$ Chêne-Bourg, Geneva, Switzerland. 


\section{References}

Arnold SE, Hyman BT, Flory J, Damasio AR, van Hoesen GW (1991) The topographical and neuroanatomical distribution of neurofbrillary tangles and neuritic plaques in the cerebral cortex of patients with Alzheimer's disease. Cereb Cortex 1:103-116.

Arriagada PV, Growdon JH, Hedley-White ET, Hyman BT (1992a) Neurofibrillary tangles but not senile plaques parallel duration and severity of Alzheimer's disease. Neurology 42:631-639.

Arriagada PV, Marzloff K, Hyman BT (1992b) Distribution of Alzheimer-type pathologic changes in nondemented elderly individuals matches the pattern in Alzheimer's disease. Neurology 42:1681-1688.

Ball MJ (1977) Neuronal loss, neurofibrillary tangles and granulovacuolar degeneration in the hippocampus with ageing and dementia. Acta Neuropathol (Berl) 37:111118

Berg L, McKeel DW, Miller P, Baty J, Morris JC (1993) Neuropathological indexes of Alzheimer's disease in demented and nondemented persons aged 80 years and older. Arch Neurol 50:349-358.

Bierer LM, Hof PR, Purohit D, Carlin L, Perl DP (1993) Neocortical neurofibrillary tangles correlate with dementia severity in Alzheimer's disease. Soc Neurosci Abstr 19:193.

Bouras C, Giannakopoulos P, Hof PR, Robakis NK, Surini M, Michel JP (1992) Distribution of neurofibrillary tangles and amyloid deposits in the hippocampus and the tem. poral neocortex: a study of a one year autopsy population from a geriatric hospital. Soc Neurosci Abstr 18:565.

Bouras C, Hof PR, Morrison JH (1993) Neurofibrillary tangle densities in the hippocampal formation in a non-de mented population define subgroups of patients with differential early pathologic changes. Neurosci Lett 153 : 131-135.

Braak H, Braak E (1990) Neurofibrillary changes confined to the entorhinal region and an abundance of cortical amyloid in cases of presenile and senile dementia. Acta Neuropathol (Berl) 80:479-486.

Braak H, Braak E (1991) Neuropathological stageing of Alzheimer-related changes. Acta Neuropathol (Berl) 82: 239-259.

Brion S, Plas J, Jeanneau A (1991) Maladie de Pick. Point de vue anatomo-clinique. Rev Neurol 147:693-704.

Chu CC, Flory J, Van Hoesen GW (1993) Orbitofrontal pathology in Alzheimer's disease. Soc Neurosci Abstr 19: 190

Crook T, Bartus RT, Ferris SH, Whitehouse P, Cohen GD, Gershon S (1986) Age-associated memory impairment: proposed diagnostic criteria and measures of clinical change-report of a National Institute of Mental Health work group. Dev Neuropsychol 2:261-276.

Crystal H, Dickson D, Fuld P, Masur D, Scott R, Mehler M, Masdeu J, Kawas C, Aronson M, Wolfson L (1988) Clinico-pathologic studies in dementia: nondemented sub jects with pathologically confirmed Alzheimer's disease. Neurology 38:1682-1687.

Dayan AD (1970) Quantitative histological studies on the aged human brain: I. Senile plaques and neurofibrillary tangles in normal patients. Acta Neuropathol (Berl) 16: 85-94.

Défossez A, Beauvillain JC, Delacourte A, Mazzuca M (1988) Alzheimer's disease: a new evidence for common epitopes between microtubule associated protein tau and paired helical filaments (PHF): demonstration at the electron microscope by a double immunogold labelling. Virchows Arch [Pathol Anat] 413:141-145.

De Lacoste MC, White III CL (1993) The role of cortical connectivity in Alzheimer's disease pathogenesis: a re. view and a model svstem. Neurobiol Aging 14:1-16

Delacourte A, Flament $S$, Dibe EM, Hublau $P$, Sablonnière B, Hémon B, Scherrer V, Défossez A (1990) Patholog ical proteins tau 64 and 69 are specifically expressed in the somatodendritic domain of the degenerating cortical neurons during Alzheimer's disease: demonstration with a panel of antibodies against tau proteins. Acta Neuropathol (Berl) 80:111-117.

Dickson DW, Crystal HA, Mattiace LA, Masur DM, Blau AD, Davies P, Yen SH, Aronson MK (1991) Identification of normal and pathological aging in prospectively studied nondemented elderly humans. Neurobiol Aging 13:179189

Fallet-Bianco C, Roudier M, Lamour Y, Davous P (1990) Etude neuropathologique de 50 cas de démence sénile. Rev Neurol 146:687-696.

Folstein MF, Folstein SE, McHugh PR (1975) Mini-mental state: a practical method for grading the cognitive state of patients for the clinician. J Psychiatry Res 12:189-198.

Gallyas F (1971) Silver staining of Alzheimer's neurofi. brillary changes by mean of physical development. Acta Morphol Acad Sci Hung 19:1-8.

Giannakopoulos P, Hof PR, Michel JP, Bouras C (1993) Quantitative immunohistochemical analysis of the dis. tribution of neurofibrillary tangles and senile plaques in the cerebral cortex of nonagenarians and centenarians Acta Neuropathol (Berl) 85:602-610.

Globus JH (1927) The Cajal and Hortega glia staining methods. A new step in the preparation of formaldehyde fixed material. Arch Neurol Psychiatry 18:263-271.

Gordon B (1992) Memory systems and their disorders. In: Diseases of the nervous system-clinical neurobiology (Asbury AK, McKhann GM, McDonald WI, eds), pp 703717. Philadelphia: Saunders.

Guntern R, Bouras C, Hof PR, Vallet PG (1992) An im proved thioflavine $S$ method for neurofibrillary tangles and senile plaques in Alzheimer's disease. Experientia 48:8-10.

Hauw JJ, Vignolo P, Duyckaerts C, Beck H, Forette F, Henry JF, Laurent M, Piette F, Sachet A, Berthaux P (1986) Etude neuropathologique de 12 centenaires: la fréqu ence de la démence sénile de type Alzheimer n'est pas particulièrement élevée dans ce groupe de personnes très agées. Rev Neurol 142:107-115

Hodges JR, Salmon DP, Butters N (1992) Semantic memory impairment in Alzheimer's disease: failure of access or degraded knowledge. Neuropsychologia 30:301-314.

Hof PR, Morrison JH (1990) Quantitative analysis of a vulnerable subset of pyramidal neurons in Alzheimer's dis ease: II. Primary and secondary visual cortex. J Comp Neurol 301:55-64

Hof PR, Morrison JH (1991) Neocortical neuronal subpopulations labeled by a monoclonal antibody to calbin din exhibit differential vulnerability in Alzheimer's dis ease. Exp Neurol 111:293-301.

Hof PR, Bouras C, Constantinidis J, Morrison JH (1990a) Selective disconnection of specific visual association pathways in cases of Alzheimer's disease presenting with Balint's syndrome. J Neuropathol Exp Neurol 49:168184

Hof PR, Cox K, Morrison JH (1990b) Quantitative analvsis of a vulnerable subset of pyramidal neurons in Alzhei. mer's disease: I. Superior frontal and inferior temporal cortex. J Comp Neurol 301:44-54.

Hof PR, Cox K, Young WG, Celio MR, Rogers J, Morrison JH (1991) Parvalbumin-immunoreactive neurons in the neocortex are resistant to degeneration in Alzheimer's disease. J Neuropathol Exp Neurol 50:451-462.

Hof PR, Bierer LM, Perl DP, Delacourte A, Buée L, Bouras C, Morrison JH (1992) Evidence for early vulnerability of the medial and inferior aspects of the temporal lobe in an 82-vear-old patient with preclinical signs of de. mentia-regional and laminar distribution of neurofi brillary tangles and senile plaques. Arch Neurol 49:946953.

Hof PR, Nimchinsky EA, Celio MR, Bouras C, Morrison JH (1993) Calretinin-immunoreactive neocortical inter neurons are unaffected in Alzheimer's disease. Neurosci Lett 152:145-149.

Hof PR, Bouras C, Perl DP, Morrison JH (1994) Quanti. 
tative neuropathologic analysis of Pick's disease cases: cortical distribution of Pick bodies and coexistence with Alzheimer's disease. Acta Neuropathol (Berl), in press.

Hubbard BM, Fenton GW, Anderson JM (1990) A quantitative histological study of early clinical and preclinical Alzheimer's disease. Neuropathol Appl Neurobiol 16:111121.

Hyman BT, Van Hoesen GW, Kromer LJ, Damasio AR (1986) Perforant pathway changes in the memory impairment of Alzheimer's disease. Ann Neurol 20:472-481.

Hyman BT, Kromer LJ, Van Hoesen GW (1988) A direct demonstration of the perforant pathway terminal zone in Alzheimer's disease using the monoclonal antibody Alz50. Brain Res 450:392-397.

Hyman BT, Van Hoesen GW, Damasio AR (1990) Memory related systems in Alzheimer's disease: an anatomic study. Neurology 40:1721-1730.

Katzman R, Terry R, DeTeresa R, Brown T, Davies P, Fuld P, Renbing X, Peck A (1988) Clinical, pathological, and neurochemical changes in dementia: a subgroup with preserved mental status and numerous neocortical plaques. Ann Neurol 23:138-144.

Kim KS, Miller DL, Sapienza VG, Chen CJ, Vai C, Grundke Iqbal I, Curry JR, Wisniewski HM (1988) Production and characterization of monoclonal antibodies reactive to synthetic cerebrovascular amyloid peptide. Neurosci Res Commun 2:121-130.

Lewis DA, Campbell MJ, Terry RD, Morrison JH (1987) Laminar and regional distribution of neurofibrillary tan gles and neuritic plaques in Alzheimer's disease: a quan titative study of visual and auditory cortices. J Neurosci 7:1799-1808.

Lippa CF, Hamos JE, Pulaski-Salo D, Degennaro LJ, Drach. man DA (1992) Alzheimer's disease and aging: effects on perforant pathway perikarya and synapses. Neurobiol Aging 13:405-411

Mann DMA, Brown AMT, Prinja D, Jones D, Davies CA (1990)A morphological analysis of senile plaques in the brains of non-demented persons of different ages using silver, immunocytochemical and lectin histochemical staining techniques. Neuropathol Appl Neurobiol 16:17-25.

Mirra SS, Heyman A, McKeel D, Sumi SM, Crain BJ, Brownlee LM, vogel FS, Hughes JP, van Belle G, Berg L (1991) The Consortium to Establish a Registry for Alzheimer's Disease (CERAD). Part II. Standardization of the neu ropathologic assessment of Alzheimer's disease. Neurol ogy 41:479-486.

Mirra SS, Hart MN, Terry RD (1993) Making the diagnosis of Alzheimer's disease. A primer for practicing patholo. gists. Arch Pathol Lab Med 117:132-144.

Morris JC, McKeel DW Jr, Storandt M, Rubin EH, Price JL, Grant EA, Ball MJ, Berg L (1991) Very mild Alzheimer's disease: informant-based clinical, psychometric, and pathologic distinction from normal aging. Neurology 41 469-478.

Morrison JH, Lewis DA, Campbell MJ, Huntley GW, Benson DL, Bouras C (1987) A monoclonal antibody to non phosphorylated neurofilament protein marks the vulnerable cortical in Alzheimer's disease. Brain Res 416:331336.

Mountjoy CQ, Roth M, Evans NJR, Evans HM (1983) Cortical neuronal counts in normal elderly controls and de. mented patients. Neurobiol Aging 4:1-11.

Mutrux S (1947) Diagnostic différentiel histologique de la maladie d'Alzheimer et de la démence sénile-pathophobie de la zone de projection corticale. Mschr Psychiat Neurol 113:100-117.

Pearson RCA, Esiri MM, Hiorns RW, Wilcock GK, Powell TPS (1985) Anatomical correlates of the distribution of the pathological changes in the neocortex in Alzheimer's disease. Proc Natl Acad Sci USA 82:4531-4534.

Price JL, Davis PB, Morris JC, White DL (1991) The distribution of tangles, plaques and related immunohistochemical markers in healthy aging and Alzheimer's dis. ease. Neurobiol Aging 12:295-312.
Rogers J, Morrison JH (1985) Quantitative morphology and regional and laminar distributions of senile plaques in Alzheimer's disease. J Neurosci 5:2801-2808.

Senut MS, Roudier M, Davous P, Fallet-Bianco C, Lamour Y (1991) Senile dementia of the Alzheimer type: is there a correlation between entorhinal cortex and dentate gy. rus? Acta Neuropathol (Berl) 82:306-315.

Squire LR, Zola-Morgan S (1991) The medial temporal lobe memory system. Science 253:1380-1386.

Suzuki WA, Zola-Morgan S, Squire LR, Amaral DG (1993) Lesions of the perirhinal and parahippocampal cortices in the monkey produce long-lasting impairment in the visual and tactile modalities. J Neurosci 13:2430-2451.

Terry RD, Peck A, DeTeresa R, Schechter R, Horoupian DS (1981) Some morphometric aspects of the brain in senile dementia of the Alzheimer type. Ann Neurol 10:184192.

Terry RD, Masliah E, Salmon DP, Butters N, DeTeresa R, Hill R, Hansen LA, Katzman R (1991) Physical basis of cognitive alterations in Alzheimer's disease: synapse loss is the major correlate of cognitive impairment. Ann Neurol 30:572-580.

Tomlinson BE, Blessed G, Roth M (1968) Observations on the brains of non-demented old people. J Neurol Sci 7:331-356.

Tomlinson BE, Blessed $G$, Roth M (1970) Observation on the brains of demented old people. J Neurol Sci 11:205242.

Ulrich J (1985) Alzheimer changes in non-demented pa. tients younger than sixty-five: possible early stages of Alzheimer's disease and senile dementia of Alzheimer type. Ann Neurol 17:273-277.

Vallet PG, Guntern R, Hof PR, Golaz J, Delacourte A, Robakis NK, Bouras C (1992) A comparative study of histological and immunohistochemical methods for neurofibril. lary tangles and senile plaques in Alzheimer's disease. Acta Neuropathol (Berl) 83:170-178.

Vermersch P, Frigard B, David JP, Fallet-Bianco C, Delacourte A (1992) Presence of abnormally phosphorylated tau proteins in the entorhinal cortex of aged non. demented subjects. Neurosci Lett 144:143-146.

Vickers JC, Delacourte A, Morrison JH (1992) Progressive transformation of the cytoskeleton associated with nor. mal aging and Alzheimer's disease. Brain Res 594:273278.

Wilcock GK, Esiri MM (1982) Plaques, tangles and dementia-a quantitative study. J Neurol Sci 56:343-356.

Zola-Morgan S, Squire LR (1984) Preserved learning in monkeys with medial temporal lesions: sparing of motor and cognitive skills. J Neurosci 4:1072-1085.

Zola-Morgan S, Squire LR, Amaral DG, Suzuki WA (1989) Lesions of perirhinal and parahippocampal cortex that spare the amygdala and hippocampal formation produce severe memory impairment. J Neurosci 9:4355-4370. 